\title{
La personalidad de las sociedades. Descripción y dinámica
}

\section{Personality of societies. Decription and dynamics}

\author{
Salvado Amigó. \\ Departament de Personalitat, Avaluació i \\ Tractaments Psicològics \\ Universitat de València \\ Valencia, Spain \\ Salvador.Amigo@uv.es
}

\author{
Antonio Caselles. \\ IASCYS member \\ Departament de Matemàtica Aplicada \\ Universitat de València \\ Valencia, Spain \\ Antonio.Caselles@uv.es
}

\author{
Joan C. Micó. \\ Institut Universitari de Matemàtica Multidisciplinar \\ Universitat Politècnica de València \\ Valencia, Spain \\ jmico@mat.upv.es
}

\begin{abstract}
La Extraversión es considerada como la dimensión fundamental y básica de la personalidad desde la Teoría del Rasgo Único de Personalidad [1]. La Extraversión, como dimensión temperamental, engloba dimensiones cognitivas como la racionalidad y la emocionalidad. La teoría de los tipos de Carl Jung [2] establece una dinámica compleja entre estas dimensiones, a partir de la cual se obtiene una tipología de la personalidad. Amigó [3] adaptó la teoría de Jung para aplicarla a las sociedades. Así, al igual que los individuos, las sociedades poseen una determinada personalidad y pueden también ser clasificadas según una tipología de personalidad. Se reflexiona sobre la historia como la dinámica de las sociedades a lo largo del tiempo, que parte de la idea de Jung de las dimensiones opuestas que se complementan e imprimen movimiento, y de la teoría de la supervivencia de las sociedades de Amigó [4], que sostiene que las crisis provocan cambios socioeconómicos que tienen a perpetuarse a través de la estructura ideológica resultante. Finalmente, se propone una metodología para aplicar la matemática de la teoría de sistemas a esta nueva concepción de la personalidad individual y social.
\end{abstract}

Keywords-Factor General de Personalidad; Extraversión; Carl Jung; Teoría de la supervivencia de las sociedades

Abstract - Extraversion is considered as the fundamental and basic dimension of personality from the Unique Trait Personality Theory [1]. Extraversion, as temperamental dimension, involves cognitive dimensions such as racionality and emotionality. Carl Jung's theory of types [2] states a complex dynamics among these dimensions. From this theory a typology of personality arises. Amigó [3] adapted Jung's theory to applye it to societies. A reflexion about history is given by the dynamics of societies through time. This approach starts from Jung's idea of the opposite dimensions that complement and imprint movement, and from Amigó's theory about societies survivance [4], which argues that crises cause socio-economic changes that have to perpetuate themselves through the resulting ideological structure. Finally, a methodology is proposed to apply the mathematics of systems theory to this new conception of the individual and social personality 


\section{La extraversión como dimensión clave de la personalidad}

Los términos extraversión e introversión fueron introducidos en el ámbito de estudio por primera vez por Carl Jung en su obra Los tipos psicológicos [2]. Estos términos fueron retomados por Eysenck en los años 50, demostrando la importancia de la dimensión de Extraversión con experimentos y estudios taxonómicos [5] para, posteriormente, en la década de los 60 y 70 desarrollar los cuestionarios EPI y EPQ, en los que la dimensión de extraversión es fundamental, como lo ha seguido siendo en todos los modelos factoriales contemporáneos [6]. Actualmente, y sobre todo influido por el modelo biológico de la personalidad de Eysenck, la extraversión se considera un dimensión temperamental de primer orden.

Para Jung los extravertidos están interesados en el mundo exterior, son abiertos y sociales, mientras que los introvertidos enfocan su interés hacia su interior, y son más reservados. Las dos actitudes son opuestas pero complementarias, las dos se compensan mutuamente. Algo parecido se desprende del modelo de Eysenck, ya que para este autor el término extraversión es en realidad la representación de un continuo extraversiónintroversión y, por tanto, representa dos polos extremos de una única dimensión y, en cierta manera, opuestos pero relacionados. Posteriormente, Amigó [1] propuso la Extraversión como la dimensión central y fundamental que aglutina y representa toda la personalidad, y que se situaría en la cumbre de la jerarquía de dimensiones de personalidad. Esta superdimensión se conoce también como Rasgo Único de Personalidad o Factor General de Personalidad [7]. La existencia de esta dimensión está avalada por una intensa investigación que ha desembocaba en un modelo sistémico complejo [8,9].

La teoría de los tipos de personalidad de Jung nos permite establecer una dinámica entre las dimensiones temperamentales y cognitivas. Así, el temperamento vendría dado por la dimensión Extraversión-Introversión, que representa la dirección de la energía psíquica: hacia el exterior en el caso de la Extraversión y hacia el interior en el caso de la Introversión. En cuanto a las dimensiones cognitivas, Jung contempla dos de ellas: Racional (pensamiento y emoción), que tiene que ver con los juicios y toma de decisiones de los individuos, e Irracional (sensación e intuición), que tiene que ver con la forma en que se percibe la información.

Basándose, por una parte, en las teorías de personalidad desde la perspectiva factorialista $y$, por otra parte, en el esquema de Jung, Amigó [3] propuso un nuevo modelo de personalidad. La dimensión temperamental es la Extraversión-Introversión (E-I), mientras que las dos dimensiones de Jung se reducen a una: RazónEmoción/Intuición, que podemos simplificar en la dimensión Razón-Emoción (R-E). Esta dimensión cognitiva agrupa las dos dimensiones cognitivas de Jung, y considera que la Emoción (también la Intuición) no es un proceso racional, como defendía Jung, sino irracional.

Con estas modificaciones, el nuevo modelo de personalidad quedaría representado en el siguiente gráfico de dos ejes:

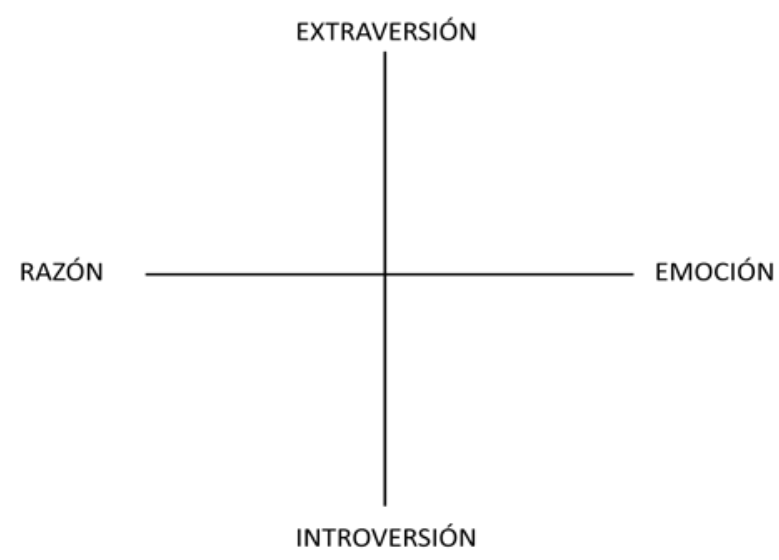

En algunos estudios se ha comprobado que hay una relación entre estas dos dimensiones. Así, la Extraversión está positivamente relacionada con la Intuición (en nuestro caso, el equivalente a la Emoción) [10]. Siendo así, el Factor General de Personalidad (FGP), que considera la superdimensión E-I como la cúspide de la jerarquía de dimensiones de personalidad, sigue siendo acertada, ya que el resto de las dimensiones, como las dimensiones cognitivas, están integradas en el modelo del FGP.

Ahora bien, a la hora de explicar el comportamiento complejo del ser humano y, en particular, su dinámica, considerar las dos dimensiones de forma separada puede ser más interesante.

En este sentido, Jung también ofrece un mecanismo de interrelación entre las dimensiones temperamentales y cognitivas. Según este autor, existe una función dominante, que representa a la persona (la imagen social del ser humano), que integra ambas dimensiones (por ejemplo, extravertido racional) y una función inferior (por ejemplo, introvertido intuitivo) opuesta a la dominante, y que constituye la sombra (el inconsciente individual). Entre estas funciones (y otras que considera Jung) se establece una dinámica que explica el funcionamiento complejo del ser humano. 
Partiendo de su teoría y de la modificación realizada por Amigó [3], las dos dimensiones representan tendencias de personalidad estables, pero que se interrelacionan de forma dinámica. Así, si bien desde la perspectiva nomotética de la Psicología podemos afirmar que los extravertidos son más emocionales que los introvertidos. A nivel idiográfico podemos encontrar todas las variaciones que nos permita la combinación de las dos dimensiones, que son cuatro: extravertido-racional, extravertido-emocional, introvertido-emocional.

Si una persona tiene la extraversión-razón como función dominante, su función inferior será la introversiónemoción, que complementa la función principal, dando como resultado un equilibrio de fuerzas psicológico. Ahora bien, en una situación crítica o de estrés agudo, es probable que emerja con más determinación la sombra, es decir, la función inferior. Incluso es posible que durante un tiempo, la función inferior ocupe el lugar de la función dominante, para, más adelante y una vez superada la crisis, volver a su posición original. Veámoslo en el siguiente diagrama:

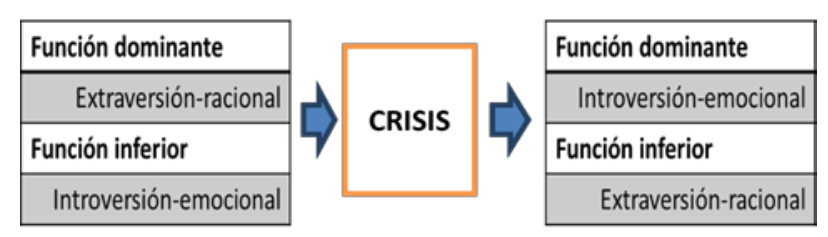

También lo podemos ilustrar con un sencillo ejemplo:

Un profesional del marketing, extravertido-racional, está organizando un equipo de trabajo para diseñar una campaña de publicidad. Su planificación de los detalles de la campaña no está siendo bien comprendida por los miembros del equipo e, incluso, algunos se muestran irresponsables en sus funciones. En este caso, el organizador puede echar mano de algunas de sus habilidades “ocultas" y emplear más recursos emocionales y/o intuitivos, atendiendo en mayor medida las motivaciones e intereses de los miembros de su equipo (extraversión-emocional). Pero también puede mantenerse rígido y llevar la situación a un límite de elevado estrés. En este caso, la función inferior puede emerger, y esta persona puede necesitar tomarse un tiempo de descanso, de relax, para meditar y estar solo (introvertidoemocional).

Este ejemplo nos hace pensar en la idea de "agotamiento" de las habilidades o los recursos personales para conseguir una meta, y la necesidad de cambiar de estrategia. Una teoría en boga hoy en día sobre el autocontrol defiende que la mente emplea recursos limitados y que el autocontrol favorece el agotamiento de esos recursos. En un estudio pionero, Baumeister et al. [11] pusieron a prueba esta hipótesis. A los participantes en un estudio se les mostraron dos platos de comida, uno con galletas y el otro con rábanos. A un grupo de ellos se les dijo que podían comer las galletas y al otro grupo que solo podían comer los rábanos. Posteriormente debían realizar un difícil rompecabezas. Los que comieron los rábanos desistieron de completar el rompecabezas a los 8 minutos, mientras que los que comieron las galletas aun perseveraban en el intento a los 19 minutos. Los investigadores dedujeron que los que comieron rábanos y, por tanto, resistieron la tentación de comer las galletas, agotaron sus recursos psicológicos en mayor medida que los demás, lo cual se plasmó en un menor tiempo de dedicación a la tarea mental de resolver el rompecabezas. Posteriormente, otros autores han obtenido resultados similares [12,13].

Así, los cambios de dirección en nuestro comportamiento, o bien la sustitución de una tendencia racional a una tendencia emocional, pongamos por caso, después de una situación de estrés, puede ser el resultado de un "agotamiento" de los recursos personales para hacer frente a esa demanda y buscar nuevas alternativas en un intento del organismo por adaptarse al ambiente.

Esto nos lleva también a una reflexión ciertamente inquietante, y es que las relaciones humanas pueden estar regidas, en gran parte, por este mecanismo de cambio basado en el "agotamiento" de los recursos. Buscamos un buen empleo, las personas más atractivas como pareja, la fama o reconocimiento, etc. Pero todo esto son recursos limitados. Nuestras relaciones sociales reflejan claramente la pugna, competencia, por esos recursos limitados, determinando nuestras aspiraciones, conflictos, envidias, y luchas con nosotros mismos.

\section{Personalidad y drogas}

Nuestro grupo de investigación ha profundizado intensamente el estudio de la relación entre el llamado Factor General de Personalidad (FGP), equivalente a la dimensión E-I, y el efecto de drogas [8,9,14]. Así, los E tenderán a reaccionar a las drogas con más excitación, en condiciones de reposo, que los I, además de inhibir el efecto más rápidamente. Esto favorece la repetición de la experiencia y, por ende, predispone a la adicción a largo plazo.

Ahora bien, con el nuevo modelo de personalidad ampliado que se presenta aquí, es posible entender mejor 
la relación entre personalidad y drogas.

Pensemos en una persona diagnosticada de trastorno por déficit de atención. Su función dominante será la extraversión emocional. En este caso, emocional sería equivalente a impulsiva y con poca adecuación social al medio. Si se le administra a esta persona metilfenidato (un estimulante), su función inferior puede emerger con claridad, y convertirse en introvertido racional, al menos por un tiempo, con un trato social más adecuado (racional).

Por otro lado, el introvertido racional que consume una dosis suficiente de alcohol, puede experimentar un cambio hacia la extraversión emocional, sintiéndose más desinhibido, sociable y más atrevido, dejándose llevar más por los impulsos.

\section{La personalidad de las sociedades}

Amigó [3] propone aplicar su modelo de personalidad de los individuos a las sociedades, tomando la personalidad social como una propiedad emergente de una sociedad en un momento histórico determinado. Para ello, Amigó incorpora a este modelo la teoría de la supervivencia de las sociedades [4], que considera que los grandes cambios socio-económicos e ideológicos de una sociedad son resultado de crisis de supervivencia.

Así se puede entender la historia de occidente, como la evolución dinámica de su personalidad. Cada sociedad, en un determinado momento histórico, tendrá una función dominante y otra inferior. Tras una situación de crisis de supervivencia, la función inferior ocupará el lugar de la función dominante hasta una nueva crisis, en la que se invertirá el orden de los factores.

Podemos, por tanto, describir las sociedades como compuestas por dos dimensiones complementarias: Extraversión-Introversión y Razón-Emoción/Intuición.

Empecemos por exponer los rasgos que caracterizan a cada uno de los polos de las dos dimensiones:

EXTRAVERSIÓN: modernidad, progreso, sociedad más abierta al cambio y al mundo.

INTROVERSIÓN: conservadurismo, sociedad más cerrada, tradicional, autárquica, proteccionista.

EMOCIONAL: idealismo, predominio de las emociones y los sentimientos, espontaneidad.

RACIONAL: realismo, predominio del pensamiento, organización y planificación.
En principio, cabría esperar que, al igual que con la personalidad individual, las sociedades extravertidas sean más emocionales y las introvertidas más racionales. Pero lo que encontrarnos, al menos en la historia de occidente, es que las sociedades más cerradas (introversión) son más emocionales/intuitivas, ya que las emociones e intuiciones representan aspectos más estáticos y contemplativos. Por su parte, las sociedades abiertas (extraversión) son más racionales, en el sentido que organizan los procesos productivos y la convivencia de la población para favorecer el progreso. Pero, también, como sucede con la personalidad de los individuos, en un momento histórico determinado podemos encontrar cualquiera de las 4 combinaciones de las dos dimensiones, dependiendo de las condiciones iniciales (historia previa).

Partiendo de estas premisas, podemos ahora exponer las características básicas que surgen de la combinación de las dos dimensiones:

EXTRAVERSIÓN EMOCIONAL: idea de progreso basada fundamentalmente en la iniciativa individual, liberalismo.

EXTRAVERSIÓN RACIONAL: búsqueda de pactos y armonía internacional, libre mercado internacional.

INTROVERSIÓN EMOCIONAL: ideal nacionalista, romanticismo, conflicto con otras naciones.

INTROVERSIÓN RACIONAL: orden, burocracia, planificación y proteccionismo.

Esto nos remite, hasta cierto punto, a la estructura bidimensional de los valores culturales de Schwartz [15], compuesta por dos dimensiones bipolares básicas de orden superior: apertura al cambio vs conservadurismo y autorrealización vs autotrascendencia [16].

En la siguiente gráfica podemos ver la estructura bidimiensional más desarrollada:

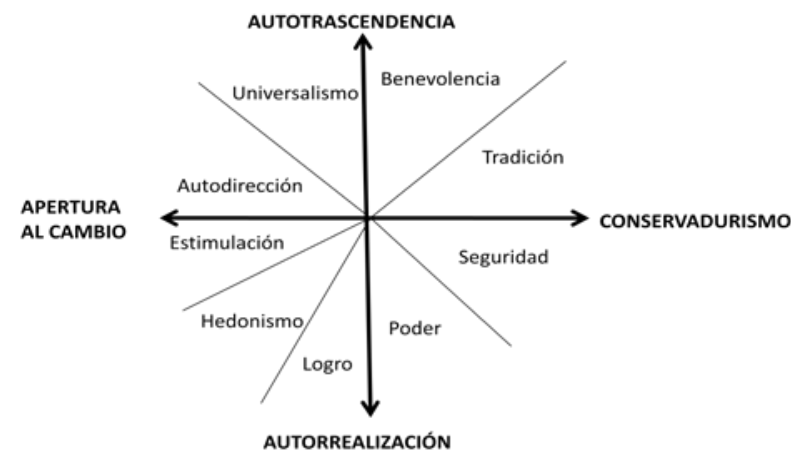


Podemos observar cómo, con nombres algo diferentes, las dos estructuras, la de Schwartz y la presentada aquí, contienen elementos muy similares integrados en una estructura bidimensional.

Veamos cómo quedan las dimensiones E-I y R-E en una gráfica y vamos a escribir en los cuadrantes los sistemas económico-sociales que les corresponde considerando la historia más reciente de occidente.

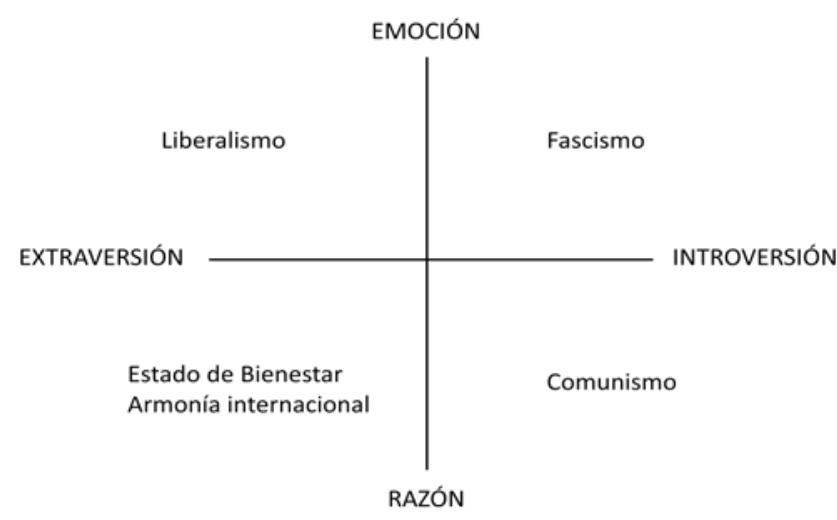

Podemos ver cómo la extraversión, que implica la apuesta por el progreso y el cambio, puede ser racional o emocional. El sistema basado en la extraversión racional se basa en la idea de facilitar el progreso a través de la organización de un Estado de Bienestar que promueva la paz social en el interior y la búsqueda de pactos internacionales para garantizar la paz y armonía con el exterior. Es el caso de la moderna Unión Europea. Por su parte, el sistema basado en la extraversión emocional apuesta por una sociedad poco organizada que potencie al máximo la iniciativa individual. Aquí la idea de progreso se basa en dejar al individuo la máxima libertad de acción. Es el caso del liberalismo, fundamentalmente, de los países anglosajones, como EEUU y Reino Unido.

En cuanto a la introversión, la sociedad introvertida racional apuesta por el orden y la estabilidad y el desarrollo socio-económico basado en la planificación. Sería el caso del comunismo soviético. En cuanto a las sociedades introvertidas emocionales, el orden y la estabilidad se consiguen a base de someterse a liderazgos particulares de "salvapatrias", cuyos designios se siguen incondicionalmente por buena parte de la sociedad. Son las dictaduras fascistas.

Vemos claramente que podemos elaborar una definición operativa de la IDEOLOGÍA de una cultura a partir de las premisas que estamos manejando. La ideología de una sociedad es una combinación de las dimensiones de Extraversión-Introversión y Razón-Emoción, en un momento dado de su historia. Si estas dimensiones cambian con el tiempo será que la ideología está también sufriendo modificaciones.

Pero nos interesa aquí desarrollar una teoría de la dinámica del cambio social a partir de las dos dimensiones que estamos considerando. Vamos para ello a retomar un importantísimo período histórico que marcó la historia de Europa y del mundo, y que analizamos en un artículo anterior [17]. Se trata de la Revolución Francesa y el cambio que trajo consigo: del Antiguo Régimen al Nuevo Sistema.

El sistema de gobierno del Antiguo Régimen era el Despotismo Ilustrado, con el poder absoluto del rey, que emanaba directamente de Dios. El orden social estaba constituido por estamentos estancos donde el ascenso de las clases bajas era inviable. A esta época histórica corresponde el estilo artístico del Barroco. Así, los aspectos emocionales (arte barroco, fundamento religioso del poder, nacionalismo) eran preeminentes en la supraestructura ideológica del Antiguo Régimen.

A partir de aquí podemos deducir fácilmente cual es la personalidad del Antiguo Régimen en términos de las dos dimensiones que estamos considerando: INTROVERSIÓN EMOCIONAL.

En cuanto al Nuevo Régimen, con un sistema de gobierno basado en la República Constitucional, una nación abierta al mundo, un sistema económico liberal, ideales de progreso y la razón como bandera, identifican esta nueva etapa surgida de la Revolución Francesa. El neoclasicismo y academicismo es el nuevo estilo artístico de la época.

También podemos deducir, a partir de esta información, qué personalidad define al nuevo sistema: EXTRAVERSIÓN RACIONAL.

La crisis demográfica y económica del Antiguo Régimen, junto a la pujanza de la burguesía y los nuevos aires de libertad que llegaban de las colonias de América, con la Declaración de Independencia de los Estados Unidos en 1775, condujeron a la Revolución Francesa de 1789. Como ya quedó expuesto anteriormente [17], la teoría de la supervivencia de las sociedades predice que en respuesta a una grave crisis socio-económica, surgirá un nuevo orden social y una nueva ideología que lo sustentará. Esta nueva ideología es la Ilustración, con su ideario de igualdad, universalidad y progreso sustentado en la razón.

Vamos ahora a aplicar el mismo esquema que anteriormente nos sirvió para entender el cambio individual al cambio social, el cambio de una época, a partir de la nueva concepción dinámica de la personalidad. 
Con toda esta información, podemos proponer un mecanismo de cambio social desde el Antiguo Régimen hasta el Nuevo Régimen surgido de la Revolución Francesa, que ilustra el siguiente diagrama:

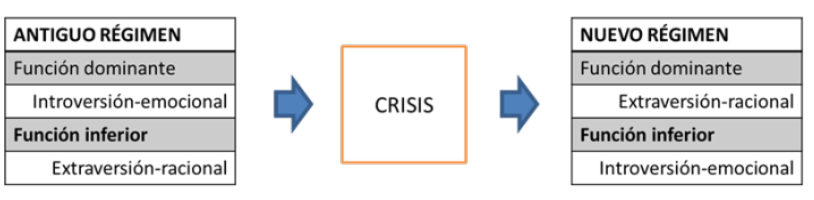

Podemos observar cómo la función dominante de la personalidad del Antiguo Régimen es la INTROVERSIÓN EMOCIONAL, y cómo la función inferior es la opuesta, es decir, la EXTRAVERSIÓN RACIONAL, como una corriente de pensamiento y acción que, poco a poco, va creciendo y tomando fuerza, oponiéndose al Antiguo Régimen. Producto de la crisis, se produce el cambio (Revolución Francesa) y la EXTRAVERSIÓN RACIONAL pasa a ser la función dominante. Con el tiempo, una nueva corriente de pensamiento y acción irá tomando fuerza y oponiéndose al nuevo sistema, de forma que, tras una nueva crisis, surgirá el Romanticismo, con una función dominante INTROVERTIDA EMOCIONAL. Y así sucesivamente.

De lo expuesto podemos deducir, tal como hicimos en el caso del cambio individual y en relación con la hipótesis de los recursos limitados del autocontrol, que las sociedades "agotan" sus recursos de subsistencia e ideológicos ante situaciones críticas (estrés), lo que conduce a cambios sociales y económicos en nuevas direcciones. La teoría de la supervivencia de las sociedades [4] ya anunciaba estos mecanismos. En el presente artículo se concreta la dirección que la supraestructura ideológica tomará en respuesta a la crisis.

Si bien, como tuvimos ocasión de comprobar cuando abordamos el cambio individual, la dinámica puede ser más compleja, este sencillo esquema nos sirve para ilustrar el mecanismo básico del cambio. Pero, no obstante, tengamos en cuenta que la función dominante puede no ser sustituida completamente por la función inferior, lo que dependerá, entre otros factores, de la intensidad de la crisis y de las condiciones iniciales (la historia previa en una determinada sociedad). Un análisis detallado de la secuencia de cambios sociales por períodos históricos breves, nos dará una idea más detallada y aproximada de la dinámica que se está produciendo.

\section{Propuesta de modelo dinámico de la personalidad social}

Ahora bien, a la hora de operacionalizar esta propuesta, de forma que pueda ser estudiada empíricamente, y así diseñar un modelo dinámico matemático, sería conveniente empezar por cuantificar productos sociales que puedan, con cierta facilidad, representar una de las dos dimensiones. Por ejemplo, la dimensión RAZÓNEMOCIÓN a partir de la producción artística, del predominio de lo racional y/o emocional de los estilos artísticos que coexistan en un determinado período histórico.

Pongamos por caso la arquitectura. Podemos concretar qué aspectos se consideran más racionales y cuales más emocionales. Así:

Racionalidad en la arquitectura: predominio de líneas rectas y figuras geométricas, espacios prácticos, pocos adornos en fachadas e interiores, estilo convencional, etc.

Emocionalidad en la arquitectura: predominio de líneas curvas, edificios poco prácticos pero llamativos, profusión de adornos, diseños arriesgados y provocadores, etc.

A partir de estos criterios, es posible cuantificar el número de construcciones arquitectónicas importantes en una época histórica, y anotar cuántas de ellas cumplen en mayor medida los requisitos para ser consideradas obras racionales o emocionales.

Si cuantificamos las obras por años, podemos seguir con mucha precisión la evolución y dinámica del estilo arquitectónico de toda una época. Lo mismo podemos hacer con otras producciones artísticas, como pintura, escultura, también con la literatura, etc., y así establecer varios subsistemas.

Si además queremos hacer lo mismo con la dimensión EXTRAVERSIÓN-INTROVERSIÓN, podemos seguir las leyes que regulan el comercio, los valores sociales y culturales de la población a través de cuestionarios, tal como hacen autores como Shwartz, Triandis, etc., estableciendo también varios subsistemas. Obviamente, también podemos considerar la influencia mutua entre los sistemas y subsistemas que representan a estas dimensiones.

Con el fin de construir el modelo dinámico propuesto es necesario tener en cuenta la estructura matemática de los estímulos que producen las respuestas descritas, tanto en individuos como en sociedades. Por ejemplo, para el caso de las drogas en los individuos, ese estímulo ya ha sido descrito matemáticamente [8,9,14]. Sin embargo, la descripción del estímulo para las sociedades sigue siendo un reto matemático.

Amigó da la clave con el concepto de crisis [3,4]: la 
descripción cuantitativa del concepto de crisis proporcionaría el patrón matemático buscado para el estímulo en las sociedades. Pero aún se puede profundizar más desde el trabajo de Arnold J. Toynbee [18]. Toynbee suele llamar incitación al estímulo, aunque a veces lo denomina también estímulo. Es equivalente al concepto de crisis de Amigó. El provecho de este trabajo es que describe con detalle el tipo de estímulos y sus características, lo que puede ayudar a diseñar el patrón matemático de los mismos. Además, los contextualiza como detonantes no sólo de los cambios en las sociedades, sinó también del nacimiento y desaparición de civilizaciones. Por ejemplo, aparecen estímulos como la desertización, que fueron capaces de hacer emerger las tres primeras civilizaciones de la Historia: la sumeria, la egipcia y la minoica.

La desertización es sólo un caso concreto de un verdadero motor de la Historia, el estímulo de la adversidad: sólo ante la adversidad nacen, crecen, cambian y mueren las civilizaciones y las sociedades que las contienen. Es paradigmático este tipo de estímulo general, que podría sugerir una estructura matemática también general, incluso a nivel del indivíduo. ¿Y si la U-invertida descrita arriba como patrón de cambio de la extraversión respecto al estímulo de una droga estimulante fuera una reacción del organismo que se opusiera a la adversidad de la droga? Si fuera así, habría que ver la droga como un factor adverso, que compensa el organismo con un incremento del nivel de estrés, de la misma manera que las civilizaciones respondieron ante la adversidad de la desertización con un "estrés", del que carecían las sociedades primitivas, y que compensara el estímulo de la desertización. De hecho, también Amigó [4] describe el Neolítico como una respuesta a otra adversidad: la superpoblación, otro estímulo también descrito en Toynbee [18] para el cambio de la civilización helénica, que la llevó a su expansión en el mediterráneo.

En definitiva, el estímulo adversidad sinónimo de crisis, sería clave para una descripción matemática del modelo dinámico propuesto, que tendría al “estrés” o extraversión generalizada como respuesta, tanto para individuos como para sociedades.

Es factible, por tanto, elaborar y comprobar un modelo dinámico matemático del desarrollo de las sociedades y de los cambios sociales. Además, esta nueva teoría de la personalidad es capaz de integrar tanto la personalidad individual como la social, abriendo una nueva perspectiva científica para el conocimiento más profundo y completo del ser humano, tanto en su vertiente individual como social. Decía Pelechano [19] que la personalidad individual hay que estudiarla en el contexto histórico particular. En ese sentido, la nuestra es una propuesta integradora que va aún más allá, dispuesta a descubrir los mecanismos fundamentales que determinan el comportamiento humano, tanto como individuos como colectivos o sociedades.

\section{REFERENCIAS}

[1] Amigó, S. (2005). La teoría del rasgo único de personalidad. Hacia una teoría unificada de la conducta y el cerebro. Valencia: Editorial UPV.

[2] Jung, C. (1921). Los tipos psicológicos. Reedición y traducción directa del alemán Andrés Sánchez Pascual. Colección: Los Libros de Sísifo. Barcelona: Edhasa. 2008.

[3] Amigó, S. (2014). El enigma de la conciencia. Entre la razón, la intuición y el misterio. Madrid: Liber Factory.

[4] Amigó, S. (2001). La teoría de la supervivencia de las sociedades. Hacia una teoría general de la evolución de la humanidad. Promolibro : Valencia.

[5] Eysenck, H. J. (1967). The Biological Basis of Personality. Springfield, MA: C. C, Thomas

[6] Wit, J. y Revelle, W. (2009). Extraversión. En M.R. Leary y R.H. Hoyle (Eds.), Handbook of individual differences in social behavior. New York: Guildord Press. pp. 27-45.

[7] Amigó, S., Caselles, A. y Micó, J.C. (2010). General Factor of Personality Questionnaire (GFPQ). Only one factor to understand personality? Spanish Journal of Psychology, 13, 5-17.

[8] Caselles, A., Micó, J.C. y Amigó, S. (2011). Dynamics of the General Factor of Personality in response to a single dose of caffeine. Spanish Journal of Psychology, 14, 675692.

[9] Micó, J.C., Amigó, S. y Caselles, A. (2014). From the Big Five to the General Factor of Personality: a Dynamic Approach. Spanish Journal of Psychology, 17, 1-18.

[10] Allison, C. y Hayes, J. (2012). The Cognitive Style Index. Technical Manual and User Guide. United Kindom: Pearson Education.

[11] Baumeister, et al. (1998). Ego depletion: Is the active self a limited resource? Journal of Personality and Social Psychology, 74(5), 1252-1265.

[12] Gailliot, M., et al. (2007). Self-control relies on glucose as a limited energy source: Willpower is more than a metaphor. Journal of Personality and Social Psychology, 92(2), 325-336.

[13] Muraven, M., \& Baumeister, R. (2000). Selfregulation and depletion of limited resources: Does self- 
control resemble a muscle? Psychological Bulletin, 126(2), 247-259.

[14] Amigó, S., Caselles, A. y Micó, J.C. (2008). A dynamical model of extraversion. British Journal of Mathematical and Statistical Pychology, 61, 211-231.

[15] Schwartz, S.H. (1992). Universals in the context and structure of human values: Theoretical advances and empirical tests in 20 countries. En M. Zanna (Ed.), Advances in experimental social psychology, vol. 25. Orlando: Academic Press.

[16] Pelechano, V. y Luengo, M.A. (2000). Creencias, valores, narrativas de vida $y$ otros conceptos de integración. En V. Pelechano (Ed.). Psicología Sistemática de la Personalidad. Barcelona: Ariel Psicología. pp. 289-342.

[17] Amigó, S., Micó, J.C. y Caselles, A. y (2009). Society survival theory and the contemporary age: proposal of a system approach. Revista Internacional de sistemas, 16, 44-48.

[18] Toynbee, A. J. (1971). Estudio de la Historia (Compendio en tres tomos de D. C. Domerwell). Madrid: Alianza Editorial.

[19] Pelechano, V. (1996). Teorías de la personalidad. Barcelona: Ariel Psicología. 